\title{
Understanding nucleation mechanism of mefenamic acid: An examination of relation between pre-assembly structure in solution and nucleation kinetics
}

Jianhua Han ${ }^{t, t}$, Mingtao Zhang, , Mingdi Liu ${ }^{\dagger, \vartheta, \neq}$, Yumin Liu ${ }^{\dagger, t}$, Jiayin Zhang ${ }^{\dagger, t}$, Kuo Wang $^{\dagger, *}$, Weiwei Tang ${ }^{*, t, t}$ and Junbo Gong ${ }^{\dagger, t}$

†School of Chemical Engineering and Technology, State Key Laboratory of

Chemical Engineering, Tianjin University, Tianjin, 300072, People's Republic of

China

¥The Co-Innovation Center of Chemistry and Chemical Engineering of Tianjin,

Tianjin, 300072, People's Republic of China

§College of Chemistry, Nankai University, Tianjin, 300071, People's Republic of

China

$\diamond$ College of Chemistry and Chemical Engineering, Qinghai Nationalities University, Xining, 810007, People's Republic of China 


\section{S1. Solubility}

\section{S1.1 Measurement method}

The solubility of MFA crystal Form I in toluene, acetonitrile and ethanol at 278-313K was measured in a $100 \mathrm{~mL}$ jacketed crystallizer under mechanical stirring. The excess crystal Form I was added into the appropriate amount of solvent to prepare suspension, and the set temperature was kept constant by a thermostatic bath (Julabo with an uncertainty $\pm 0.05 \mathrm{~K}$. At each temperature, the solution was stirred for at least $48 \mathrm{~h}$ and then settled for another $30 \mathrm{~min}$. About $2 \mathrm{~mL}$ of clear solution was taken out, filtered through a $0.45 \mu \mathrm{m}$ preheated membrane, and diluted to determine the concentration of MFA by ultraviolet-visible (UV) absorption spectroscopy at $286 \mathrm{~nm}$ (toluene $284 \mathrm{~nm}$ ). Five different concentrations of MFA were selected to construct the UV concentration standard curve of MFA in each solvent. Before the solubility measurement, the concentration of the solution was measured under different solid-liquid equilibrium time to evaluate the appropriate equilibrium time. It was found that the concentration remained stable after $48 \mathrm{~h}$ and the suspension powders were always Form I. The solubility was measured in triplicate at each equilibrium temperature.

\section{S1.2 Solubility data of MFA}

Figure S1 shows the determined solubility data as a function of temperature in toluene, ethanol, and acetonitrile. Expectedly, the solubility of MFA increases with increasing temperature. The solubility of MFA in ethanol is evidently higher than those in toluene and acetonitrile. In addition, the measured solubility data were also compared 
with the reported values in the literature (Table S1). (Mudalip et al., 2013) Apparently, the measured solubility data in this study were in well consistent with the reported ones.

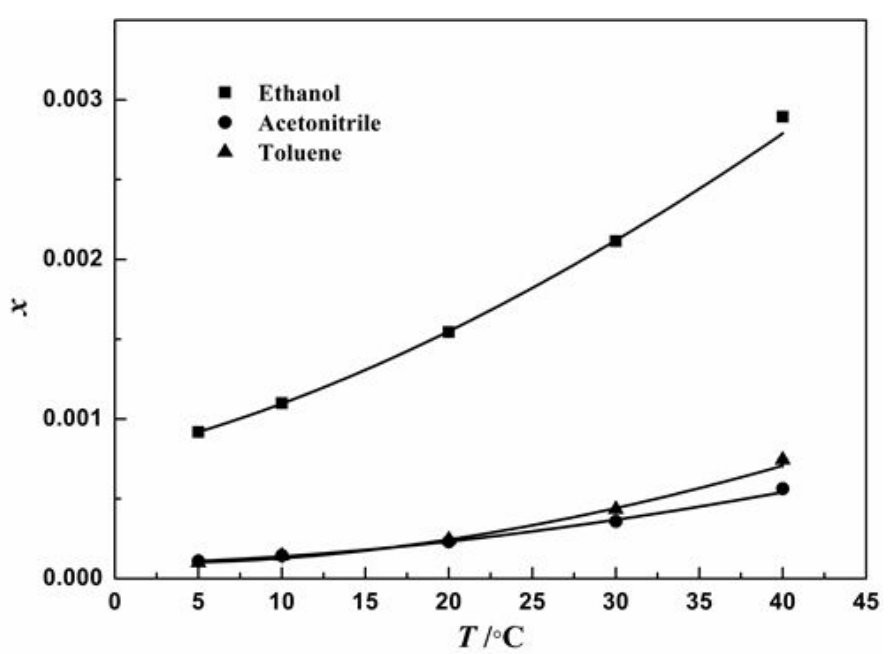

Figure S1. Temperature-dependent solubility of MFA in ethanol, acetonitrile, and toluene.

Table S1. Solubility data of MFA in ethanol measured in this work in comparisons to the literature data.

\begin{tabular}{|c|c|c|}
\hline Temperature & $x$ (sol. values $)$ & $X^{*}$ (sol lit. values $)$ \\
\hline${ }^{\circ} \mathrm{C}$ & mole fraction & mole fraction \\
\hline 20 & $0.00155\left( \pm 2 \cdot 10^{-5}\right)$ & I \\
\hline 25 & l & 0.0019 \\
\hline 30 & $0.002115\left( \pm 5 \cdot 10^{-6}\right)$ & 0.0020 \\
\hline 35 & l & 0.0024 \\
\hline 40 & $0.00289\left( \pm 3 \cdot 10^{-5}\right)$ & 0.0032 \\
\hline
\end{tabular}


S2. Nuclear magnetic resonance spectroscopy

\section{$\mathrm{S} 2.1{ }^{1} \mathrm{H}$ spectroscopy}<smiles>O=C(O)c1ccccc1Nc1cccc(I)c1F</smiles><smiles>CCCN</smiles>

$\mathrm{NH}$

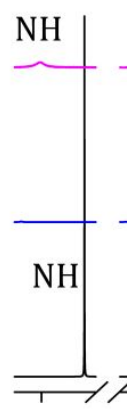

9.3

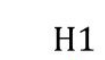

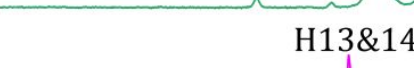

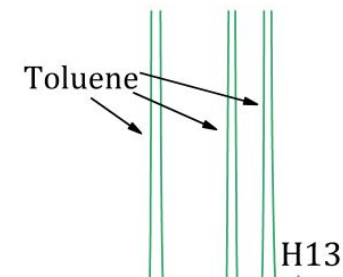

H13
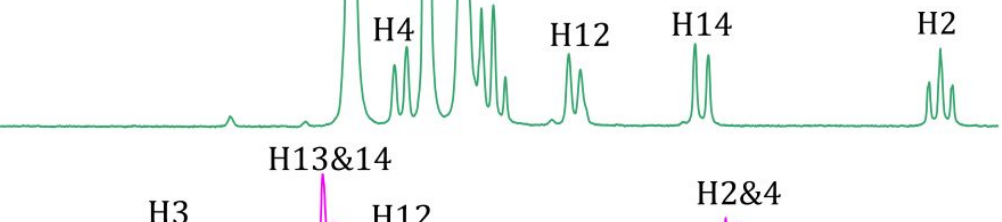

hl

H12

$\mathrm{H} 2 \& 4$
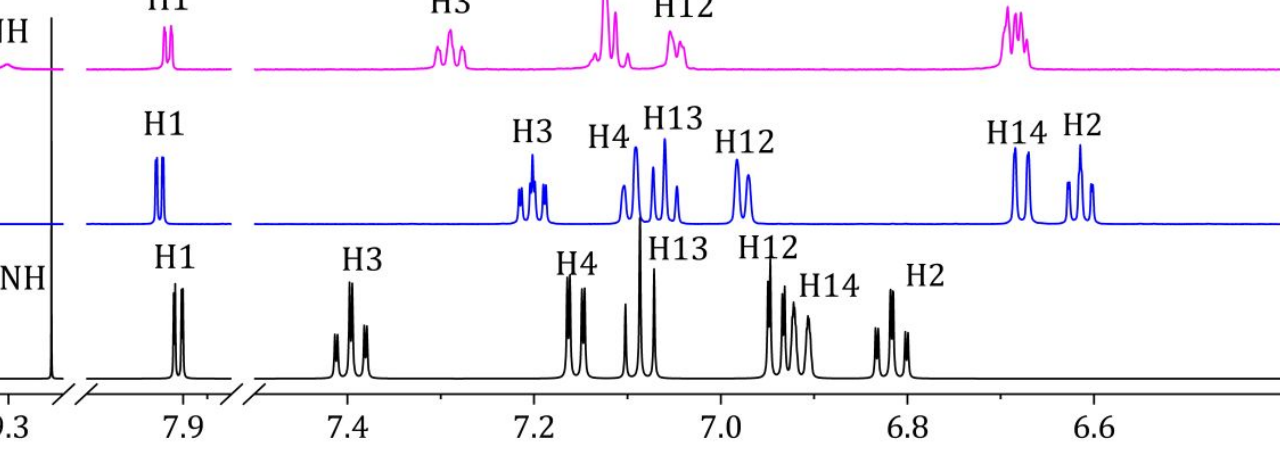

$\delta(\mathrm{ppm})$

Figure S2. Proton assignments in in ethanol (blue), acetonitrile (magenta) and toluene (olive green). The hydrogen spectrum predicted by MestReNova (black) is displayed at the bottom (Chemical shifts of $\mathrm{H}_{17}$ and $\mathrm{H}_{18}$ were located nearby 2.15 and 2.30, respectively) 


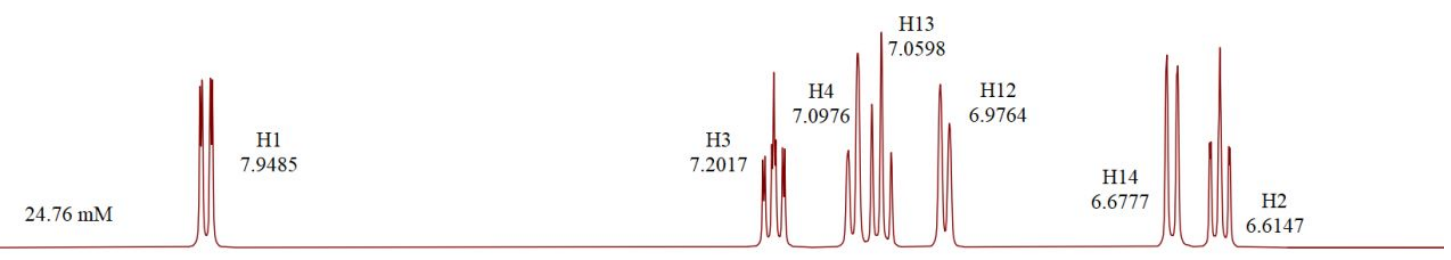

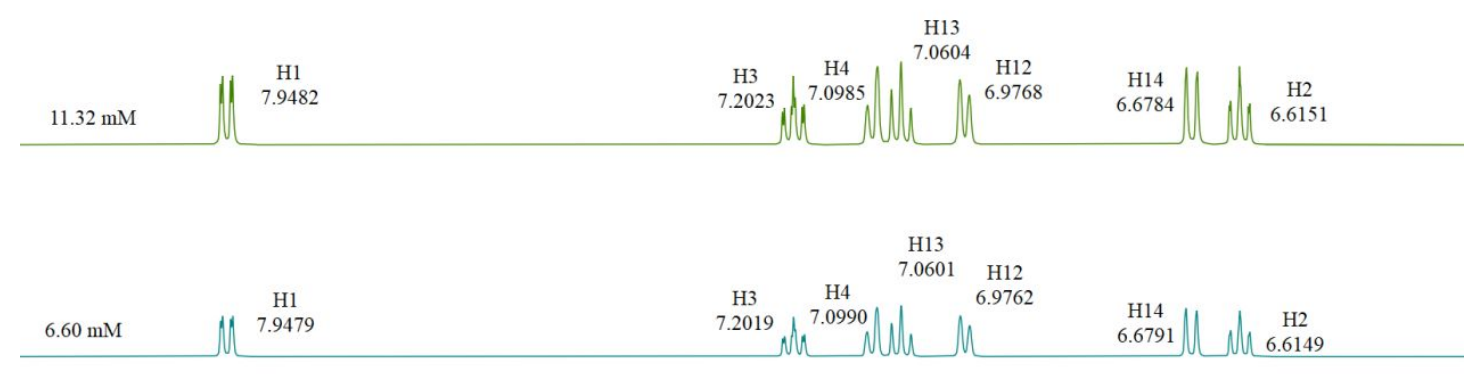

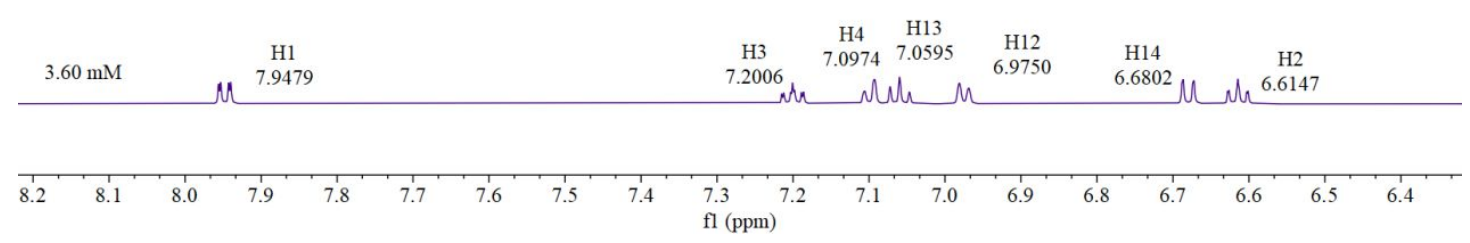

Figure S3. Concentration-dependent chemical shifts of $\mathrm{H}_{1}, \mathrm{H}_{2}, \mathrm{H}_{3}, \mathrm{H}_{4}, \mathrm{H}_{12}, \mathrm{H}_{13}$ and $\mathrm{H}_{14}$ in ethanol. ( $\mathrm{NH}$ and $\mathrm{OH}$ do not show up because they were active protons. Chemical shifts of $\mathrm{H}_{17}$ and $\mathrm{H}_{18}$ were located at 2.15 and 2.30, respectively)

S2.2 Heteronuclear Multiple Bond Correlation (HMBC) Spectroscopy 


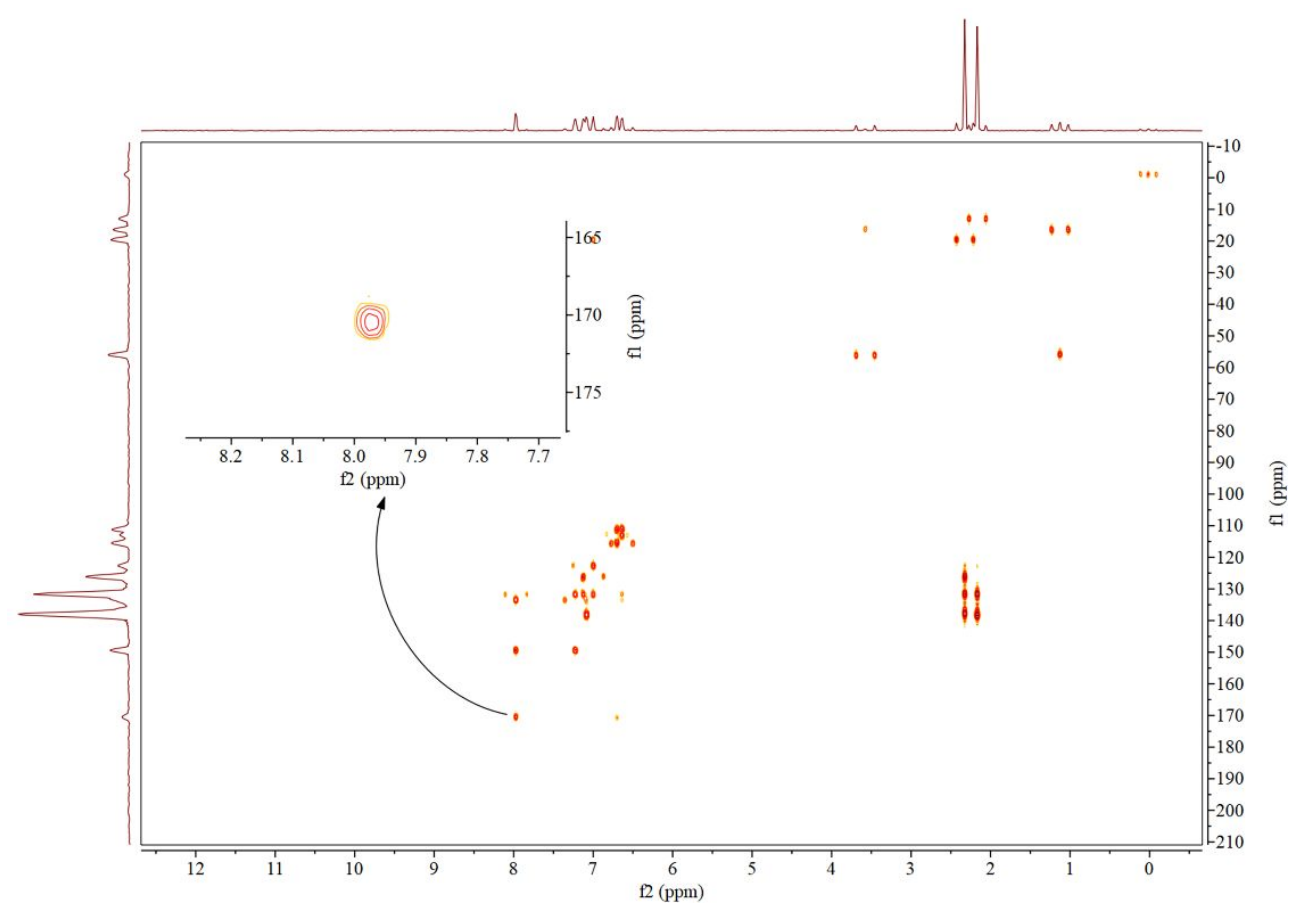

Figure S4. Typical HMBC spectrum of MFA in ethanol $(24.76 \mathrm{mM})$. The NH-C(O) correlation peak was used to determine the chemical shift of $\mathrm{C}=\mathrm{O}{ }^{13} \mathrm{C}$ resonance.

\section{S2.3 Data processing model}

S2.3.1 Combined dimerization and ionization isotherm model (CDIIM)

$$
\begin{gathered}
\delta_{o b s}=\delta \mathrm{m} \frac{C_{m}}{C_{t o t}}+\delta \frac{2 K_{d} C_{m}^{2}}{C_{t o t}}+\delta_{i} \frac{\sqrt{10^{-p k} C_{m}}}{C_{t o t}} \\
C_{t o t}=C_{m}+2 K_{d} C_{m}^{2}+\sqrt{10^{-p k} C_{m}}
\end{gathered}
$$

S2.3.2 Ionization isotherm model (IIM)

$$
\begin{gathered}
\delta_{o b s}=\delta m \frac{C_{m}}{C_{t o t}}+\delta \frac{\sqrt{10^{-p k} C_{m}}}{C_{t o t}} \\
C_{t o t}=C_{m}+\sqrt{10^{-p k} C_{m}}
\end{gathered}
$$




$$
\begin{gathered}
\delta o b s=\delta m \frac{C_{m}}{C_{t o t}}+\delta d \frac{2 K_{d} C_{m}^{2}}{C_{t o t}} \\
C_{t o t}=C_{m}+2 K_{d} C_{m}^{2}
\end{gathered}
$$

where $\delta_{o b s}$ denotes the experimental chemical shift, $\delta_{m}$ is the chemical shift of monomer, $\delta_{d}$ represents the chemical shift of dimer, $\delta_{i}$ is the chemical shift of monovalent anion, $C_{t o t}$ is the experimental concentration, and $C_{m}$ is the concentration of monomer.

\section{S2.4 Chemical shift Fitting results}

Table S2. Fitting results of ${ }^{1} \mathrm{H}$ and ${ }^{13} \mathrm{C}$ chemical shifts of MFA in ethanol, acetonitrile,

\begin{tabular}{|c|c|c|c|c|c|}
\hline \multirow{2}{*}{$\begin{array}{l}\text { Classification of } \\
\text { NMR spectrum }\end{array}$} & \multirow{2}{*}{$\begin{array}{c}\text { Data } \\
\text { processing } \\
\text { model }\end{array}$} & \multicolumn{2}{|c|}{ Fitting parameters } & \multicolumn{2}{|c|}{ Error analysis } \\
\hline & & $p K_{a}$ & $\begin{array}{c}K_{d} \\
/ \mathrm{mol}^{-1} \cdot \mathrm{L}\end{array}$ & RMSD & $\mathrm{R}^{2}$ \\
\hline $\begin{array}{c}{ }^{1} \mathrm{H} \text { chemical shifts } \\
\text { in ethanol }\end{array}$ & CDIIM & 6.81 & $5.35 \times 10^{-7}$ & $2.48 \times 10^{-3}$ & 0.88 \\
\hline $\begin{array}{c}{ }^{1} \mathrm{H} \text { chemical sifts in } \\
\text { acetonitrile }\end{array}$ & CDIIM & 5.97 & $8.89 \times 10^{-4}$ & $6.21 \times 10^{-4}$ & 0.99 \\
\hline${ }^{1} \mathrm{H}$ chemical shifts & DIM & I & 144.67 & $7.65 \times 10^{-3}$ & 0.86 \\
\hline
\end{tabular}
and toluene. 
$\mathrm{C}=\mathrm{O}{ }^{13} \mathrm{C}$ chemical

shift in ethanol

$\mathrm{C}=\mathrm{O}{ }^{13} \mathrm{C}$ chemical

shift in acetonitrile

$\mathrm{C}=\mathrm{O}{ }^{13} \mathrm{C}$ chemical

DIM

144.67

$5.97 \times 10^{-2}$

0.99

shift in toluene 
Table S3. The calculated proportion of homodimer under different supersaturations.

\begin{tabular}{ccccccc}
\hline & & $C_{M F A} \cdot 10^{-3}$ & $C_{m o n} \cdot 10^{-3}$ & $C_{\text {dim }} \cdot 10^{-3}$ & $\frac{C_{\text {dim }}}{C_{M F A}}$ & $\frac{C_{\text {dim }}}{C_{\text {mon }}+C_{\text {dim }}}$ \\
& & $/ \mathrm{M}$ & $/ \mathrm{M}$ & $/ \mathrm{M}$ & $/ \%$ & $/ \%$ \\
\hline Spontaneous & 3.40 & 10.89 & 4.65 & 3.12 & 28.67 & 40.20 \\
nucleation & 2.10 & 6.73 & 3.39 & 1.67 & 24.77 & 32.93 \\
\hline \multirow{2}{*}{ Supersaturation } & 0.77 & 2.46 & 1.66 & 0.40 & 16.22 & 19.35 \\
& 1.28 & 4.09 & 2.41 & 0.84 & 20.55 & 25.86 \\
in NMR tube & 0.51 & 1.64 & 1.21 & 0.21 & 12.98 & 14.92 \\
& 0.26 & 0.82 & 0.68 & 0.07 & 8.25 & 9.00 \\
& 0.13 & 0.41 & 0.37 & 0.02 & 4.83 & 5.08 \\
\hline
\end{tabular}

\section{S3. DFT calculations}

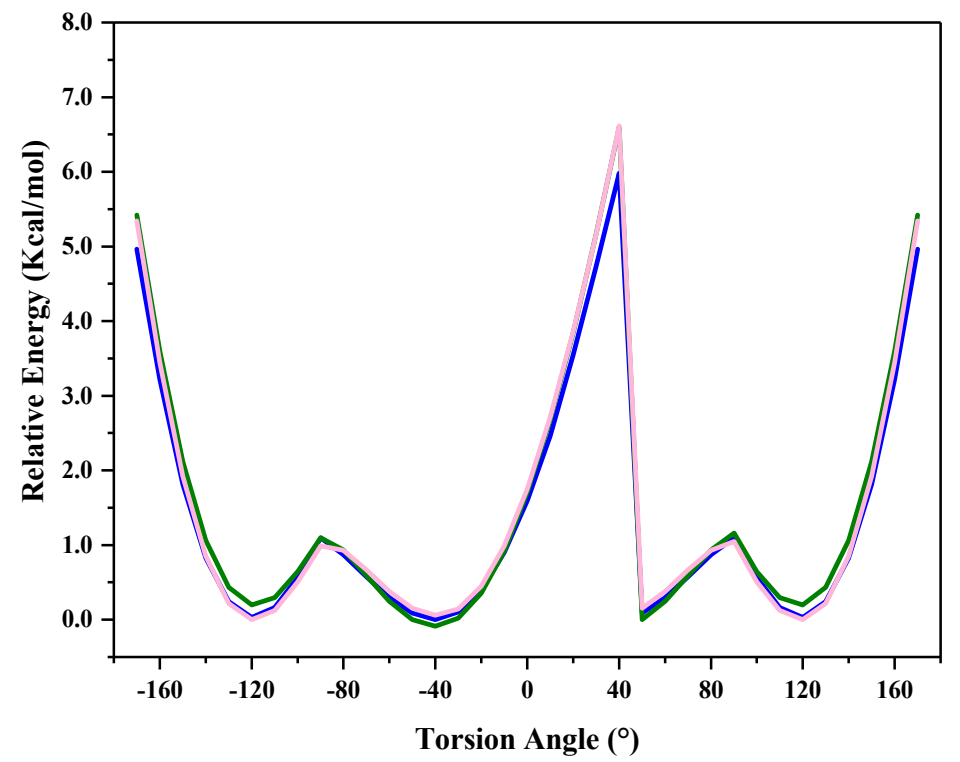

Figure S5. Relaxed potential energy surface (PES) scan in vacuum (pink), ethanol (blue), toluene (green) ethanol at B97D/6-31+g(d,p) level. 
Table S4. Relative Gibbs free energy $(\Delta G, 298 \mathrm{~K})$ of MFA monomers, solvated monomers, and dimers in toluene, acetonitrile, and ethanol.

\begin{tabular}{|c|c|c|c|c|}
\hline \multirow{2}{*}{ Solvent } & \multirow{2}{*}{ Permittivity } & \multirow{2}{*}{ Status ${ }^{[a]}$} & \multicolumn{2}{|c|}{$\Delta G / \mathrm{kJ} \cdot \mathrm{mol}^{-1[\mathrm{~b}]}$} \\
\hline & & & PL & TL \\
\hline & & M & 0.5 & 0 \\
\hline \multirow[t]{3}{*}{ Toluene } & 2 & & & \\
\hline & & $\mathrm{D}$ & -8.3 & 3.8 \\
\hline & & M & 0.4 & 0 \\
\hline \multirow[t]{2}{*}{ Acetonitrile } & 36 & M-S & 23.1 & 21.8 \\
\hline & & $\mathrm{D}$ & 2.0 & 10.1 \\
\hline \multirow[t]{3}{*}{ Ethanol } & 25 & M & 1.0 & 0 \\
\hline & & M-S & 20.4 & 24.6 \\
\hline & & $\mathrm{D}$ & 8.3 & 15.5 \\
\hline
\end{tabular}

[a]: $\mathrm{M}$ for monomer, $\mathrm{S}$ for solvent, $\mathrm{D}$ for dimer;

[b]: Gibbs free energy relative to TL conformation in standard gas phase (1 atm, 298 $\mathrm{K})$.

\section{S4. Induction time and nucleation rates}

\section{S4.1 Induction time measurements}




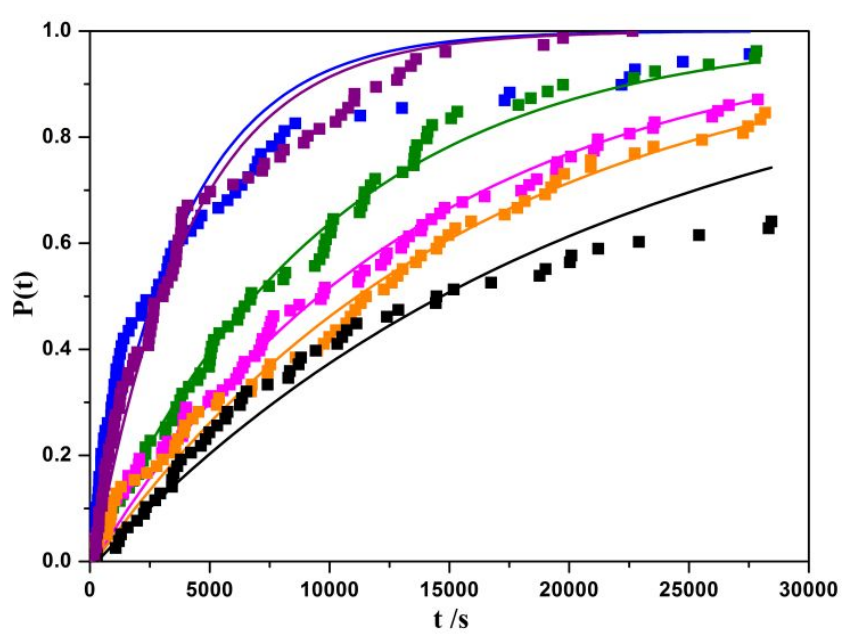

Figure S6. Experimentally determined probability distributions $P(t)$ of the induction times $(t)$ for MFA of 1.70 (black), 1.79 (orange), 1.89(magenta), 1.99 (green), 2.05 (purple), and 2.09 (blue) supersaturation ratios in ethanol at $283 \mathrm{~K}$. Solid lines are the fits to eq 2, with $t_{g}$ fixed as the fastest induction time per supersaturation ratio measured.

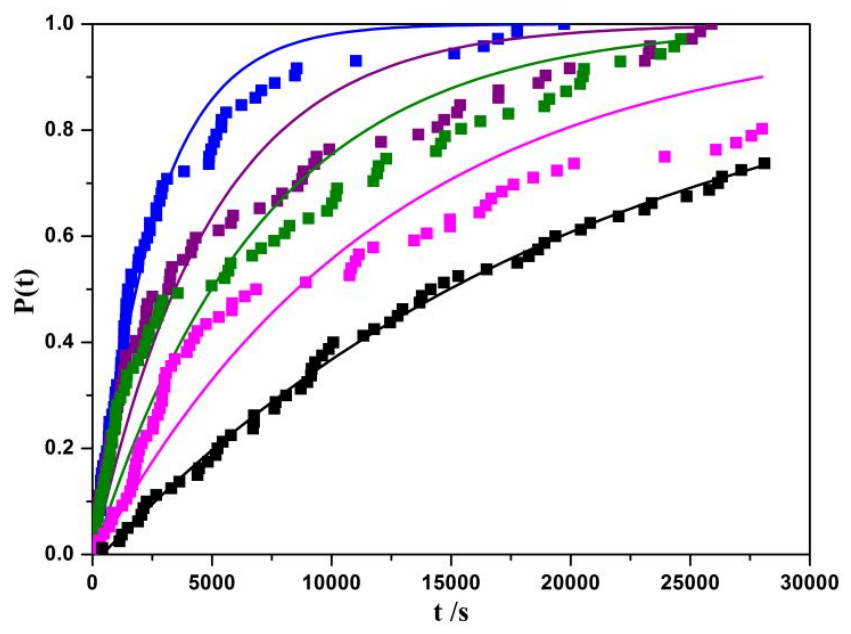

Figure S7. Experimentally determined probability distributions $P(t)$ of the induction times $(t)$ for MFA of 1.68 (black), 1.87 (magenta), 1.96 (green), 2.06 (purple), and 2.18 (blue) supersaturation ratios in ethanol at $293 \mathrm{~K}$. Solid lines are the fits to eq 2, with $t_{g}$ fixed as the fastest induction time per supersaturation ratio measured. 


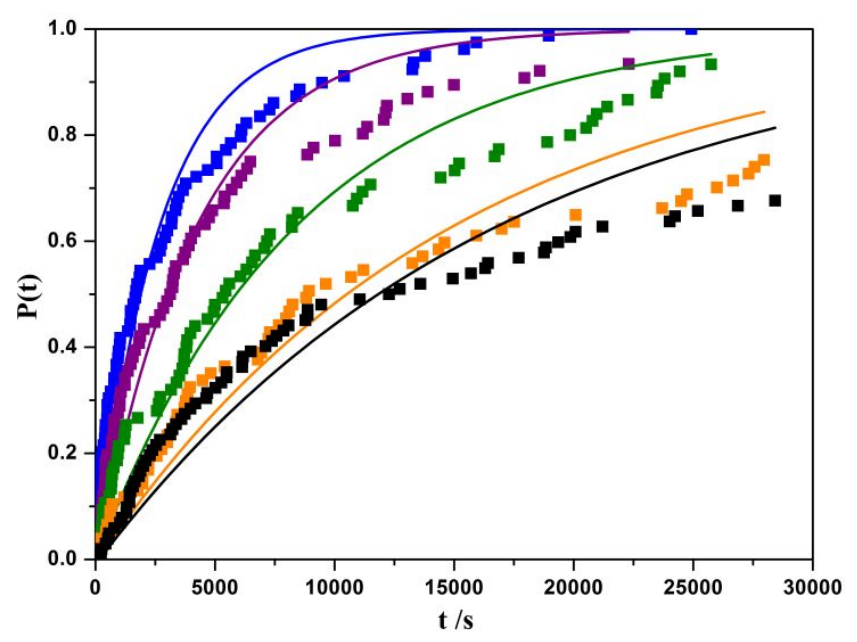

Figure S8. Experimentally determined probability distributions $P(t)$ of the induction times ( $t$ ) for MFA of 1.69 (black), 1.83 (orange), 1.88 (green), 2.04 (purple), and 2.14 (blue) supersaturation ratios in ethanol at $303 \mathrm{~K}$. Solid lines are the fits to eq 2, with $t_{g}$ fixed as the fastest induction time per supersaturation ratio measured.

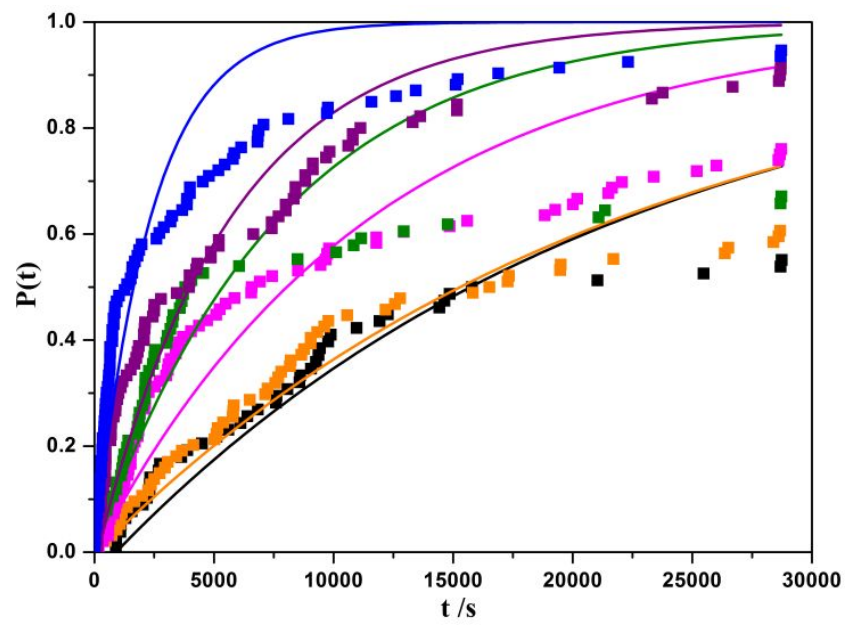

Figure S9. Experimentally determined probability distributions $P(t)$ of the induction times $(t)$ for MFA of 1.67 (black), 1.75(orange), 1.87 (magenta), 1.95 (green), 2.06 (purple), and 2.16 (blue) supersaturation ratios in acetonitrile at $293 \mathrm{~K}$. Solid lines are the fits to eq 2, with $t_{g}$ fixed as the fastest induction time per supersaturation ratio measured. 


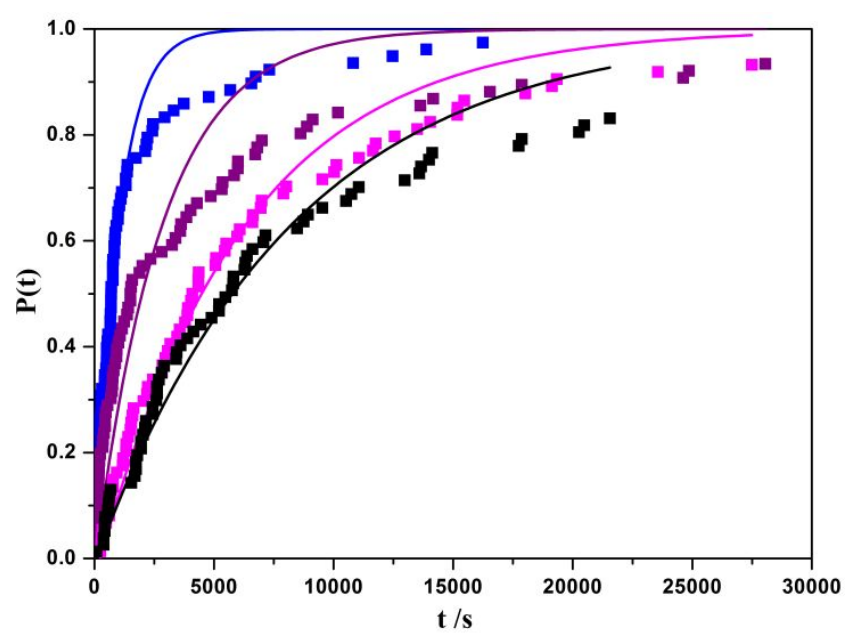

Figure S10. Experimentally determined probability distributions $P(t)$ of the induction times $(t)$ for MFA of 1.68 (black), 1.88(magenta), 2.07(purple), and 2.17(blue) supersaturation ratios in acetonitrile at $303 \mathrm{~K}$. Solid lines are the fits to eq 2 , with $t_{g}$ fixed as the fastest induction time per supersaturation ratio measured.

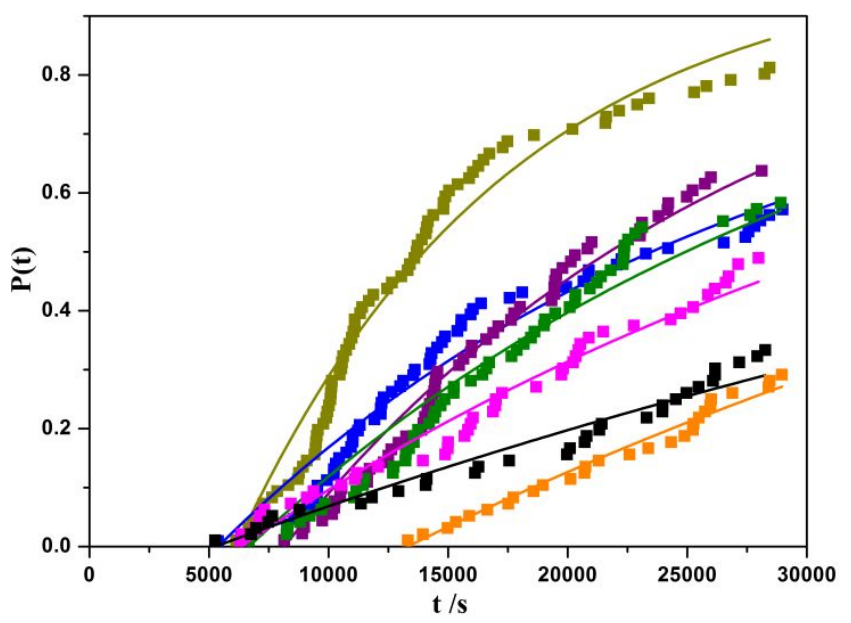

Figure S11. Experimentally determined probability distributions $P(t)$ of the induction times $(t)$ for MFA of 2.50 (black), 2.61 (orange), 2.74 (magenta) 2.86(green), 2.98 (purple), 3.14 (blue), and 3.32(reseda) supersaturation ratios in toluene at $283 \mathrm{~K}$. Solid lines are the fits to eq 2, with $t_{g}$ fixed as the fastest induction time per supersaturation ratio measured. 


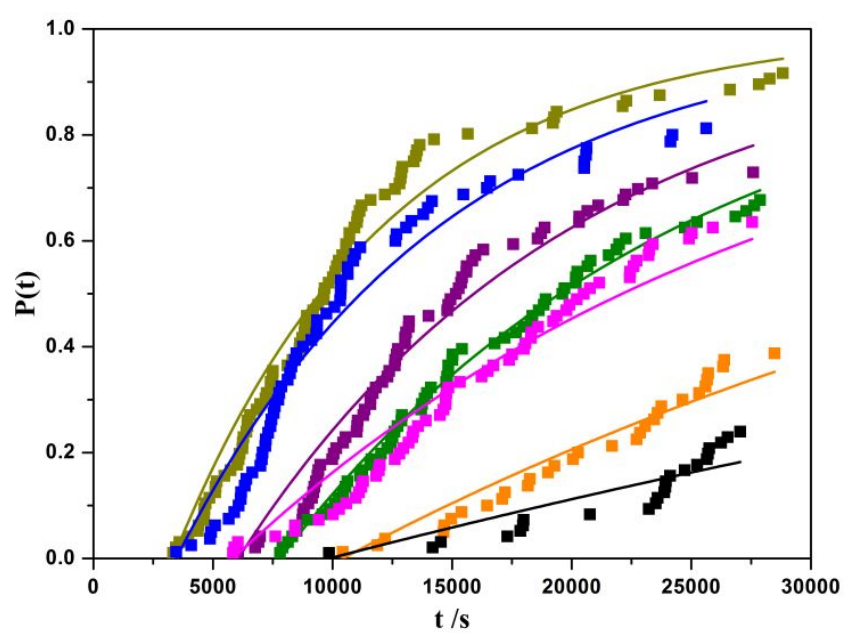

Figure S12. Experimentally determined probability distributions $P(t)$ of the induction times $(t)$ for MFA of 2.11 (black), 2.17 (orange), 2.20 (magenta) 2. 29(green), 2.46 (purple), 2.53 (blue), and 2.70(reseda) supersaturation ratios in toluene at $293 \mathrm{~K}$. Solid lines are the fits to eq 2, with $t_{g}$ fixed as the fastest induction time per supersaturation ratio measured.

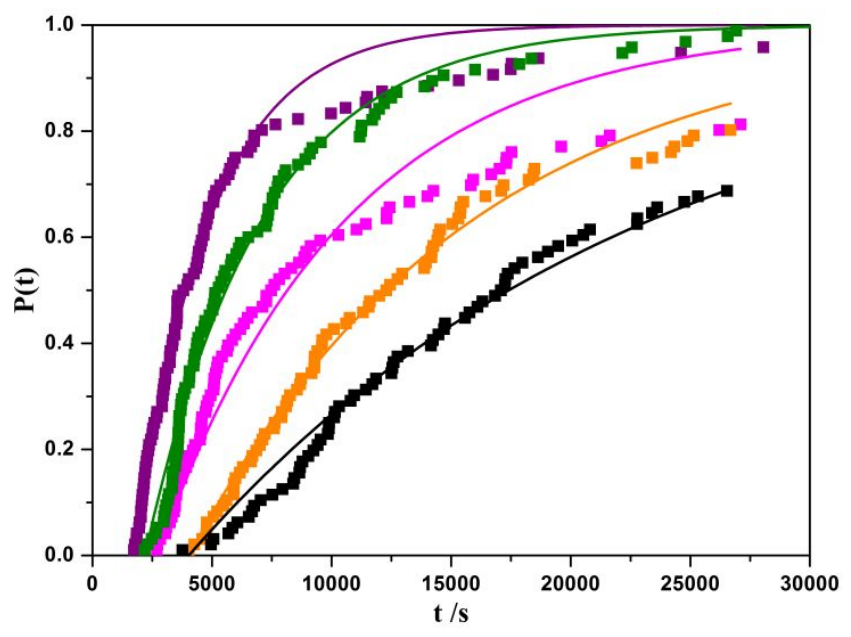

Figure S13. Experimentally determined probability distributions $P(t)$ of the induction times ( $t$ ) for MFA of 2.04 (black), 2.12 (orange), 2.21 (magenta), 2. 36(green), and 2.4652 (purple) supersaturation ratios in toluene at $303 \mathrm{~K}$. Solid lines are the fits to eq 2, with $\operatorname{tg}$ fixed as the fastest induction time per supersaturation ratio measured. 


\section{S4.2 Nucleation kinetics}

According to CNT, the nucleation rate $J$ can be written by

$$
J=f_{n}{ }^{*} X_{n}{ }^{*}-g_{n}^{*}+1 X_{n}^{*}+1=Z f_{n}{ }^{*} C_{n}^{*}
$$

in which $f_{n}{ }^{*}$ and $g_{n}{ }^{*}$ represent the frequency of attachment and detachment of monomer, respectively. $X_{n}{ }^{*}$ and $C_{n}{ }^{*}$ represent the actual nucleus concentration and the nucleus concentration that would exist in the equilibrium between the nuclei and the supersaturated solution. $z$ is the Zeldovich factor, which can be expressed by

$$
\mathrm{z}=\frac{X_{n}{ }^{*}}{C_{n}{ }^{*}}\left(1-\frac{g_{n}{ }^{*}+1 X_{n}{ }^{*}+1}{f_{n}{ }^{*} X_{n}{ }^{*}}\right)=\frac{\ln S \sqrt{k_{B} T}}{\sqrt{12 \pi \Delta G_{c}}}
$$

where $\Delta_{G_{\mathcal{C}}}$ is the free energy of crystal nucleus, $k_{B}$ is the Boltzmann constant, and

$T$ is the absolute temperature. $C_{n}{ }^{*}$ can be calculated by the following equation:

$$
C_{n}^{*}=C œ \exp \left(-\frac{\Delta G_{c}}{k B T}\right)
$$

where $C_{0}$ is the concentration of nucleation sites and can be estimate as $\frac{1}{v_{0}}$ when homogeneous nucleation occurs $\left(_{V O}\right.$ is the molecule volume of MFA in crystal Form I).

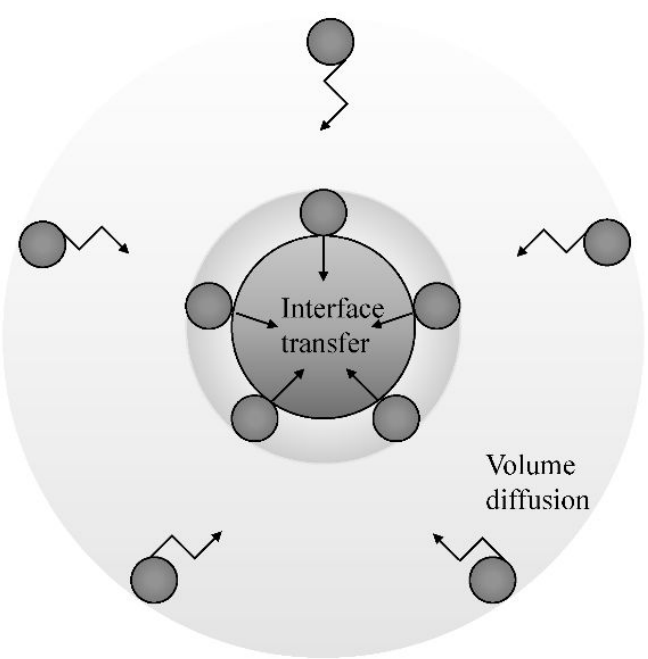


Figure S14. Interface-transfer or volume-diffusion mechanism of attachment of building units (the circles) to a spherical cluster

During the assembly of a nucleus, building units must transfer from a dissolved, solvated state to an adsorbed, partially desolvated state on the nucleus surface, which can be roughly divided in to two steps, volume diffusion and interface transfer. If the process were controlled by volume diffusion, $f_{n}{ }^{*}$ could be expressed as the product of diffusion coefficient $D$, the concentration $C$, the radius of a fictitious spherical nucleus $r^{*}$, and surface area $c\left(n^{*} V 0\right)^{2 / 3}$.

$$
f_{n}^{*}=\frac{D C c\left(n^{*} v 0\right)^{2 / 3}}{r^{*}}=\sqrt{4 \pi} c D\left(n^{*} v o\right)^{1 / 3} C_{e q} S
$$

If the process were controlled by interface transfer, $f_{n}{ }^{*}$ is expressed as the product of diffusion coefficient $D$, the concentration $C$, the adhesion coefficient $\lambda$, the molecular diameter $d o \approx\left(\frac{6 v_{0}}{\pi}\right)^{1 / 3}$, and surface area $c\left(n^{*} v 0\right)^{2 / 3}$.

$$
f_{n}{ }^{*}=\frac{D C \lambda_{c}\left(n^{*}{ }^{*}\right)^{2 / 3}}{d o}=\left(\pi_{V o l 6}\right)^{1 / 3} \lambda D c\left(n^{*}\right)^{2 / 3} C_{e q} S
$$

When the nucleation rates $J$ was expressed in a general form as eq $\mathrm{S} 12$, the kinetic parameter $A_{o}$ will be calculated by eq S13 for volume-diffusion controlled attachment mechanism or eq S14 for interface-transfer controlled mechanism.

$$
\begin{gathered}
J=A o S \exp \left(-\frac{B}{\ln ^{2} S}\right) \\
A o=\left(\frac{4 c^{3}}{27 B V O}\right)^{1 / 3} \quad D C_{e} q \ln S=A o \ln S \\
A o=\left(\frac{2 B}{6^{5} \pi_{V O}^{4}}\right)^{1 / 6} \lambda D C_{e q}
\end{gathered}
$$




\section{S4.3 Thermodynamic parameters}

According to CNT, thermodynamic parameter $B$ and the effective surface energies of crystal nucleus, $\gamma_{e f f}$, can be expressed by

$$
\begin{gathered}
B=\frac{4 c^{3} \gamma^{3} V^{2}}{27\left(k_{B} T\right)^{3}} \\
\gamma_{\text {eff }}=\frac{3 k_{B} T}{c}\left(\frac{B}{4{ }_{V O}^{2}}\right)^{1 / 3}
\end{gathered}
$$

Figure S5. Fitting results of $\ln (J / S)$ or $\ln (J / S \ln S)-1 / \ln ^{2} S$ plots in ethanol, acetonitrile, and toluene at three temperatures for two attachment mechanisms.

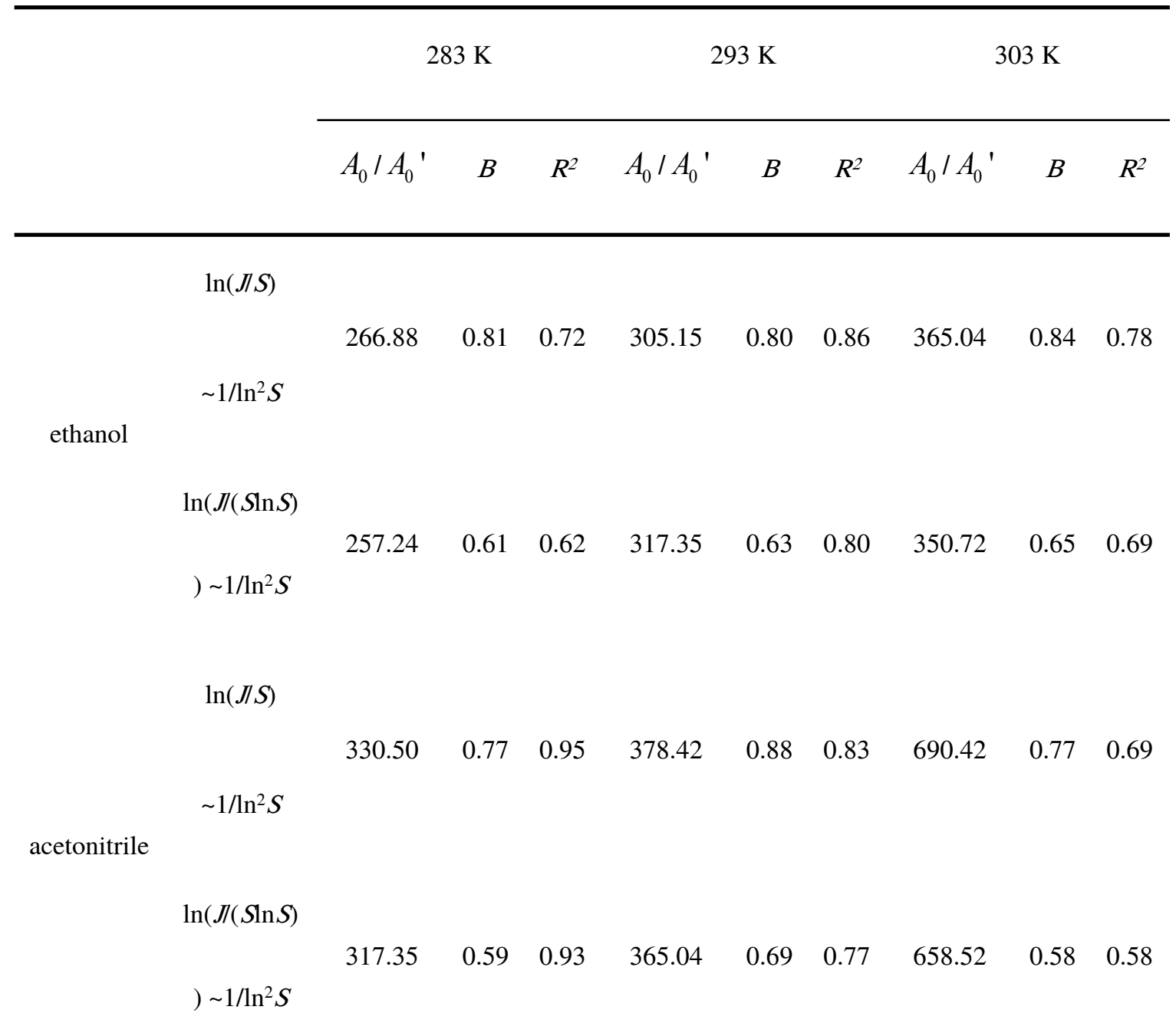


$\ln (J / S)$

$\begin{array}{lllllllll}109.95 & 2.71 & 0.92 & 361.41 & 2.25 & 0.84 & 992.27 & 2.00 & 0.99\end{array}$

$\sim 1 / \ln ^{2} S$

toluene

$\ln (J /(S \ln S)$

$\begin{array}{lllllllll}64.07 & 2.17 & 0.88 & 254.68 & 1.88 & 0.77 & 735.10 & 1.67 & 0.99\end{array}$

) $\sim 1 / \ln ^{2} S$ 